\title{
Potential Field Based Motion Planning with Steering Control and DYC for ADAS
}

\author{
Nurbaiti Wahid ${ }^{* 1}$, Hairi Zamzuri ${ }^{2}$, Nurhaffizah Hassan ${ }^{3}$, Mohd Azizi Abdul Rahman ${ }^{4}$ \\ ${ }^{1,3}$ Faculty of Electrical and Engineering, Universiti Teknologi MARA, 23000 Dungun, Terengganu, Malaysia \\ ${ }^{1,2,3,4}$ Malaysia Japan International Institute of Technology, Universiti Teknologi Malaysia, 54100 Jalan \\ Sultan Yahya Petra, Kuala Lumpur, Malaysia \\ Corresponding author, e-mail: nurbaiti@tganu.uitm.edu.my¹, hairi.kl@utm.my², \\ nurhaffizah@tganu.uitm.edu.my ${ }^{3}$, azizi.kl@utm.my ${ }^{4}$
}

\begin{abstract}
In this study, the development of motion planning and control for collision avoidance driver assistance systems is presented. A potential field approach has been used in formulating the collision avoidance algorithm based on predicted vehicle motion. Then, to realize the advanced driver assistance systems (ADAS) for collision avoidance, steering control system and direct yaw moment control (DYC) is designed to follow the desired vehicle motion. Performance evaluation is conducted in simulation environment in term of its performance in avoiding the obstacles. Simulation results show that the vehicle collision avoidance assistance systems can successfully complete the avoidance behavior without colliding.
\end{abstract}

Keywords: potential field, motion planning, steering control, DYC, ADAS

Copyright $\odot 2017$ Universitas Ahmad Dahlan. All rights reserved.

\section{Introduction}

Traffic accident prevention and collision avoidance are the critical topic being concerned in automotive world. Regarding to the statistic given by Polis Diraja Malaysia (PDRM), traffic accident and fatalities is increasing from year to year [1]. In 2013, the number of traffic accidents had been increased by $3.1 \%$ from 462423 to 477204 compared to 2012. In 2014 the number of traffic accidents is slightly decreased to $0.21 \%$ which contributed to 476196 cases. Nevertheless, the number of road crashed still retain in a high level. Since decades ago, the engraving problem caused by traffic accidents had been continually voiced out by global community. About 1.2 million annual deaths due to traffic accident around the world report by World Health Organization (WHO) in 2004 proved the problem scale [2, 3]. Therefore, the development of advanced safety technology as the advanced driver assistance systems (ADAS) for accident preventions and collision avoidance are essential.

The aim of advances safety technology is to take the surrounding of vehicle environment into account for collision prevention and reduce the traffic accidents by assist the driver during critical situation. Collision avoidance systems are one of the vehicle safety technologies under ADAS group are expected to enhance vehicle safety and reduce collision's severity. The successful collision avoidance systems are relied on the sensory system, motion planning and the actuator manipulation control to complete the avoidance maneuver. The needs for enhanced collision avoidance systems technology for ground vehicle continuously show significant challenge for the system efficiency.

Path planning and motion planning of advanced driver assistance systems are the important software elements used in vehicle navigation for collision avoidance [4]. Generally motion planning for collision avoidance can be divided into two clasifications which are global and local path planning [5]. Global path planning refers to the well-known environment while local path planning is determined if environment is unknown. It also can be classified into off-line and on-line depending on the obstacle behavior. Off-line motion planning is used to formulate the case of static obstacle where the route decision is predefined. On the contrary, on-line motion planning used to map the moving obstacle and the route decision is identified based on the dynamics environment [6]. There are numerous motion planning techniques had been used 
in automotive vehicle navigation systems for collision avoidance. Motion planning using artificial potential field was introduced by [7] and had been implemented in robotics field [8, 9] based on idea in filling the robot workspace with the information from environment. Application of potential field techniques in motion planning also had been extended into vehicle guidance systems including lane keeping [10] and obstacle avoidance [11]. In executing the behavior of obstacle avoidance, the control system is designed to ensure the desired vehicle motion is followed safely without colliding.

This study presents the motion planning and control system for vehicle collision avoidance driver assistance systems. The structure of this paper is divided into three main parts; the second part described the formulation of motion planning to identify the vehicle desired motion. This part also discussed on the control system designed based on proposed collision avoidance algorithm to track the desired trajectory. The third part concentrated on the simulation results of vehicle manuevre behaviour in avoiding the collision for a given driving scenario. The conclusion and recommendation of future works are given in the final part of this paper.

\section{Collision Avoidance Motion Planning and Control System}

This section described the motion planning and control system formulation for collision avoidance system. In this work, motion planning was formulated using artificial potential field approach while controller designed for lateral motion control was realized by steering wheel controller and DYC.

\subsection{Vehicle Collision Avoidance System Overview}

Figure 1 shows the system overview of driver assistance systems for collision avoidance. Firstly, the algorithm for collision avoidance was generated to identify desired yaw rate value, $r$ by using potential field approach.

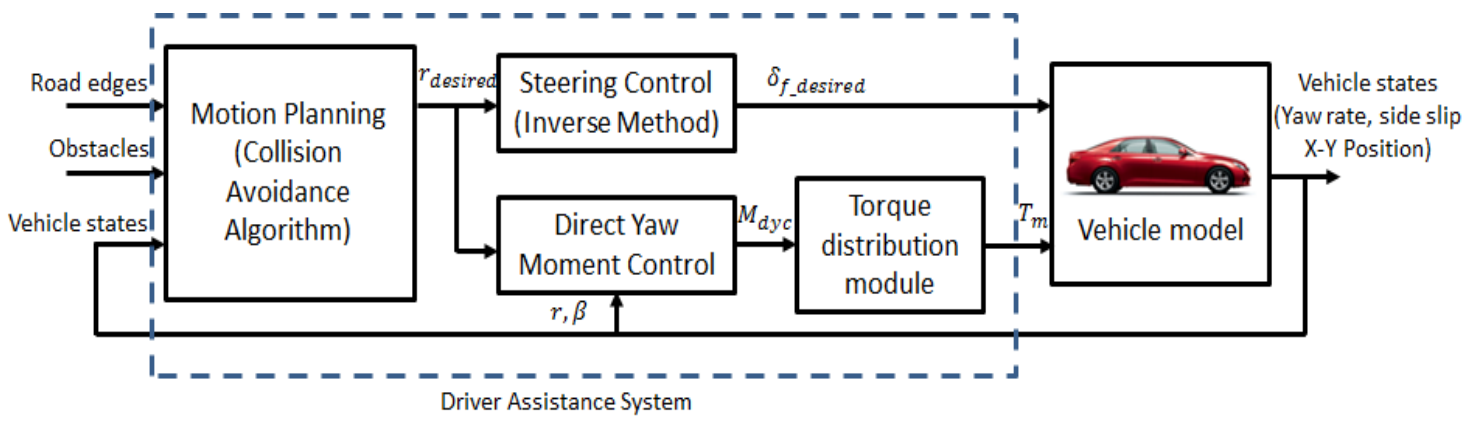

Figure 1. Collision avoidance driver assistance systems overview

\subsection{Vehicle Collision Avoidance System Overview}

In this work, potential field approach was used for motion planning in generating collision avoidance algorithm due to mathematical simplicity [12] and minimal computational effort [13]. The environmental information such as the goal to the target location and obstacle is represented as attractive and repulsive potential energy respectively [14].

In general, the goal to the target location represented by attractive potential function used to guide the vehicle to the desired destination. The road edges and the obstacles represented by repulsive potential function that keep the obstacles far away from the vehicle as well as to avoid the collision. In this work, the potential field formulation was calculated regarding to the previous works that had been done in [13-17]. Several potential functions were formulated for road edges and obstacle based on situation of two stationary obstacles located on the straight lane. The summation of all potential functions value can be denoted as Eequation (1).

$$
U_{\text {total }}=U_{\text {road }}+U_{\text {obstacles } 1}+U_{\text {obstacles } 2}
$$


Potential function formulation of road edges was used to maintain the vehicle movement on center of the lane when there is no obstacle. Each side of the lane represented the highest potential energy to prevent the vehicle from lane departure. The formulation of repulsive potential function of road edges can be expressed as following,

$$
U_{\text {road }}(X, Y)=w_{\text {road }}\left(1-\exp \left[\left(Y-Y_{c}\right)^{2} / 2 \sigma_{\text {road }}^{2}\right]\right)
$$

Where $w_{\text {road }}, \sigma_{\text {road }}$ and $Y_{c}$ represented weight, standard deviation of the repulsive potential function of the road edges and $Y$-coordinate of the road center respectively. Figure 2 shows the potential function of road edges.
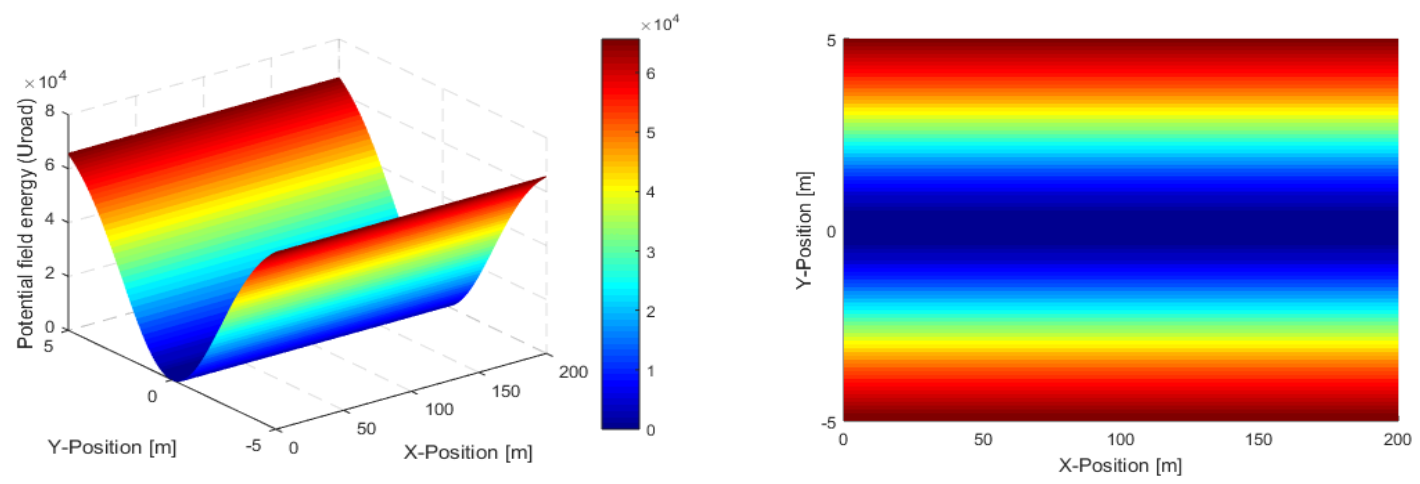

Figure 2. Repulsive potential function of road edges

The potential field function of stationary obstacles was computed to keep away the vehicle from obstacles and remain the safety distance by increasing the potential energy towards the direction of obstacles. The lane change manoeuvre was executed to avoid the obstacle and the potential function of two stationary obstacles can be formulated as Equation (3) and (4).

$$
\begin{aligned}
& U_{\text {obstacle } 1}(X, Y)=w_{\text {obstacle } 1} \exp \left(-\left[\left(X-X_{c o 1}\right)^{2} / \sigma_{c x}^{2}\right]-\left[\left(Y-Y_{c o 1}\right)^{2} / \sigma_{c y}^{2}\right]\right) \\
& U_{\text {obstacle } 2}(X, Y)=w_{o b s t a c l e 2} \exp \left(-\left[\left(X-X_{c o 2}\right)^{2} / \sigma_{c x}^{2}\right]-\left[\left(Y-Y_{c o 2}\right)^{2} / \sigma_{c y}^{2}\right]\right)
\end{aligned}
$$

The $w_{\text {obstacle } 1}$ and $w_{\text {obstacle } 2}$ both exhibits potential function weight of stationary obstacles while $\sigma_{c x}, \sigma_{c y}, \quad Y_{c 01}$ and $Y_{c 02}$ represented standard deviation of X-Y direction and X-Y coordinate of vehicle center. The potential function of two stationary obstacles is shown is Figure 3.
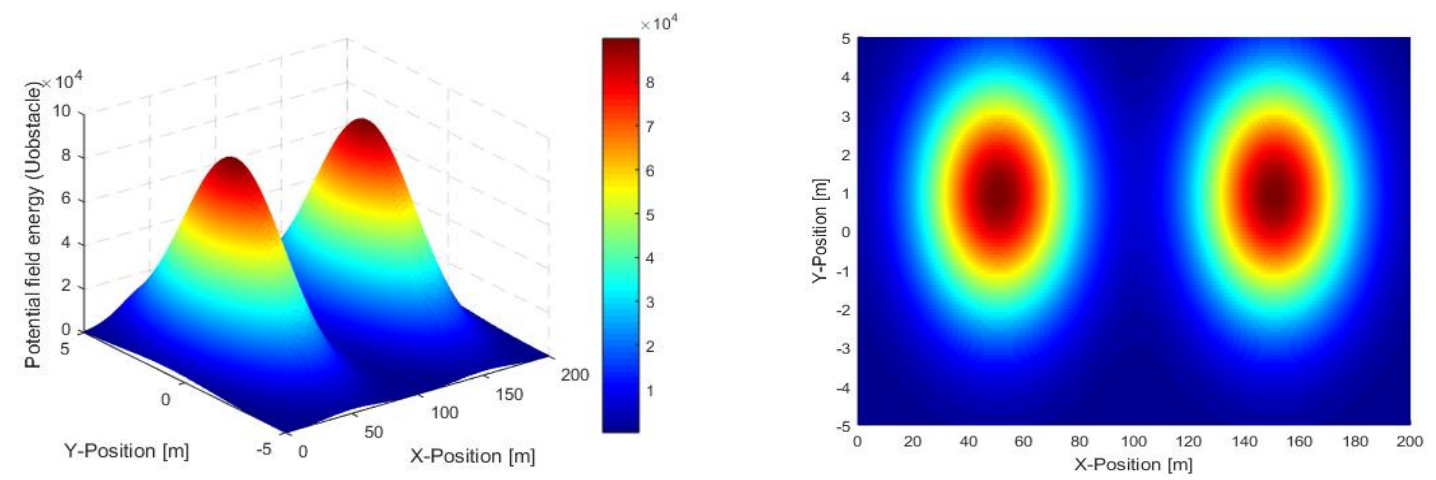

Figure 3. Repulsive potential function of stationary obstacles 
In this work, the parameters of the weight of potential function, $w_{\text {road }}, w_{\text {obstacle } 1}$, $w_{\text {obstacle } 2}$ and standard deviation of potential function, $\sigma_{\text {road }}, \sigma_{c x}$ and $\sigma_{c y}$ were used to modify the shape of potential field energy that represented the road edges and stationary obstacles. By referring to the equation (1), the total potential field energy map for road edges and obstacles is illustrated in Figure 4.

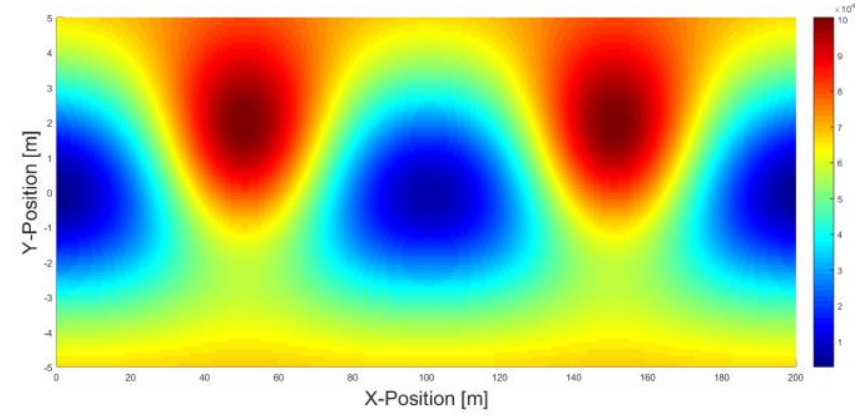

Figure 4. Map for total potential field function

In realizing the driver assistance systems, the reference yaw rate value was calculated to find the optimal performance function for motion planning based on work done in [16, 17]. The application of potential field with optimal control theory as in $[16,17]$ was implemented to replace the gradient descent method [11] that generally used in creating the motion planning. This method was used to avoid the local minima problem that might be existed during the task execution. The prediction yaw rate value which consists of several yaw rate reference candidates was computed for the range of $r_{\text {minimum }}$ and $r_{\text {maximum }}$ value and can be expressed as following.

$$
\begin{aligned}
& r_{\text {prediction }}(n)=r_{\text {minimum }}+\Delta r_{\text {prediction }} \times i \\
& i=0,1,2, \ldots, n
\end{aligned}
$$

In this work, the $r_{\text {minimum }}$ and $r_{\text {maximum }}$ both was set to $-0.5 \mathrm{rad} / \mathrm{s}$ and $0.5 \mathrm{rad} / \mathrm{s}$ respectively. The vehicle position prediction value at prediction time horizon and evaluation cost function of yaw rate reference value can be represented as equation (6) and (7) below:

$$
\begin{aligned}
& X_{\text {prediction }}(n)=X_{\text {current }}+\int_{t}^{t+t_{n}} V \cos \left(\theta+r_{\text {prediction }}(n) \times t\right) d t \\
& Y_{\text {prediction }}(n)=Y_{\text {current }}+\int_{t}^{t+t_{n}} V \sin \left(\theta+r_{\text {prediction }}(n) \times t\right) d t
\end{aligned}
$$

where $\theta$ denoted as vehicle yaw angle. By using application of optimal control theory in [17], the evaluation cost function for identification of yaw rate reference value can mathematically be expressed as following equation. The reference yaw rate was defined from the minimum value of evaluation cost function that was calculated on each sampling time where $q$ specified as weight of yaw rate prediction.

$$
J(n)=\sum\left(U_{\text {total }}\left[X_{\text {prediction }}(n), Y_{\text {prediction }}(n)\right]+q r_{\text {prediction }}^{2}\right)
$$




\subsection{Control System Formulation}

In this work the lateral motion control algorithm was designed which consist of steering control system and direct yaw moment control. This control strategy was applied in order to track the vehicle desired motion for collision prevention. The reference steering angle was determined by using linear single-track vehicle model based on inverse dynamics method in following the desired path. The state-space represented the linear vehicle model equation can be expressed as below:

$$
\dot{x}=A x+B u
$$

where,

$$
\begin{aligned}
& A=\left[\begin{array}{cc}
-\frac{2\left(C_{f}+C_{r}\right)}{m v} & -1-\frac{2\left(l_{f} C_{f}-l_{r} C_{r}\right)}{m v^{2}} \\
-\frac{2\left(l_{f} C_{f}-l_{r} C_{r}\right)}{I_{z}} & -\frac{2\left(l_{f}^{2} C_{f}+l_{r}^{2} C_{r}\right)}{I_{z} v}
\end{array}\right], B=\left[\begin{array}{cc}
\frac{2 C_{f}}{m v} & 0 \\
\frac{2 l_{f} C_{f}}{I_{z}} & \frac{1}{I_{z}}
\end{array}\right] \\
& x=\left[\begin{array}{c}
\beta \\
r
\end{array}\right], u=\left[\begin{array}{c}
\delta \\
M_{z}
\end{array}\right]
\end{aligned}
$$

By using inverse method and negligible the effect of side slip angle, the steering angle value can be calculated as in equation (10).

$$
\delta_{f}=\frac{l^{2} 2 C_{f} C_{r}+m v^{2}\left(C_{f} l_{f}-C_{r} l_{r}\right)}{2 C_{f} C_{r} l v}
$$

The desired steering angle was determined by considering the value of steering gear ratio and can be computed as follows:

$$
\delta_{f_{-} \text {desired }}=R \times \delta_{f}
$$

For direct yaw moment control, the corrective yaw moment and corrective distributed torque both were formulated as equation (12) and (13) respectively based on works in [18, 19]. $K_{m d y c 1}$ and $K_{m d y c 2}$ referred to corrective yaw moment gain, $r_{w}$ indicated the tire wheel radius and $w$ denoted as vehicle track width.

$$
\begin{aligned}
& M_{d y c}=K_{\text {mdyc1 }}\left(r_{\text {desired }}-r\right)+K_{\text {mdyc2 }}\left(\beta_{\text {desired }}-\beta\right) \\
& T_{m}=-\frac{2 M_{d y c} r_{w}}{w}
\end{aligned}
$$

With approximation of vehicle longitudinal motion as one-wheel vehicle model, the longitudinal force can be identified as following equation where $F_{r}$ indicated the vehicle running resistance.

$$
F_{x}=\frac{T_{m}}{r_{w}}-F_{r}
$$

\section{Results and Discussion}

\subsection{Simulation Driving Scenario}

In the simulation works, the driving scenario was created based on two stationary obstacles (parking vehicle) located on the straight lane as shown in Figure 5. Additionally, Table 1 tabulated the parameters value that had been set for the simulation scenario condition. The parking vehicle, both was located at coordinate $X_{c 01}$ and $X_{c 02}$ of vehicle centre where $\mathrm{Y}$ - 
coordinate was set to $Y_{c 01}$ and $Y_{c 02}$ from the road centre. For simulation works the vehicle speed was set to $50 \mathrm{~km} / \mathrm{h}$ to evaluate the performance of collision avoidance assisting system.

Table 1. Parameters value of simulation driving scenario

\begin{tabular}{ccc} 
Symbol & Variable & Value (SI unit) \\
\hline$V$ & Vehicle velocity & $50 \mathrm{~km} / \mathrm{h}$ \\
$X_{c 01}$ & Coordinate of X of stationary obstacle 1 & $49.8 \mathrm{~m}$ \\
$X_{c 02}$ & Coordinate of X of stationary obstacle 2 & $151.2 \mathrm{~m}$ \\
$Y_{c 01}$ & Coordinate of $Y$ of stationary obstacle 1 & $1 \mathrm{~m}$ \\
$Y_{c 02}$ & Coordinate of $Y$ of stationary obstacle 2 & $1 \mathrm{~m}$ \\
$Y$ & Lateral distance of road center & 0
\end{tabular}

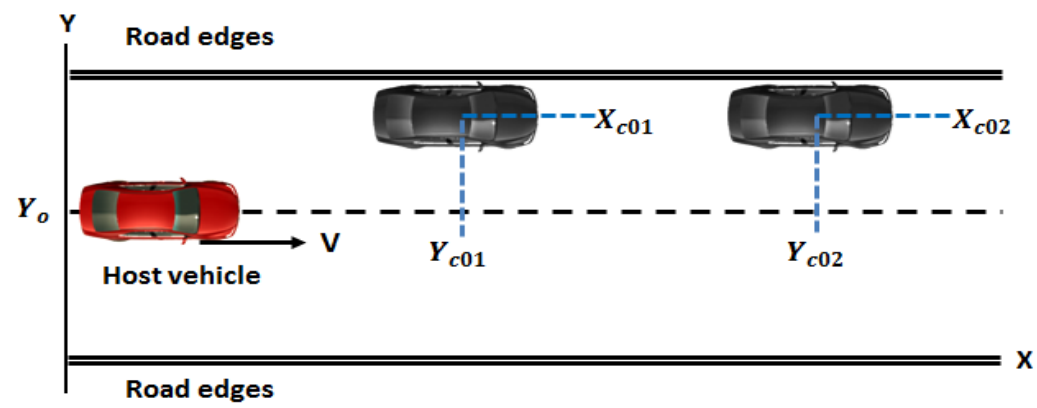

Figure 5. Simulation driving scenario

\subsection{Simulation Results}

The simulation results show the vehicle manoeuvre behaviour with regards to the lane change for collision avoidance. The road edges and stationary obstacles potential function were work together in effecting the manoeuvre behaviour as illustrated in Figure 6. From these results, it had been noticed that the host vehicle can avoid the two stationary obstacles in stable condition. By applying DYC, the vehicle speed value was diverse during the steering manoeuvre in avoiding the obstacles. The vehicle speed was reduced when the vehicle did the lane change to avoid the obstacle and start to increased again when the host vehicle went back to the original lane.

The corrective yaw moment input, yaw rate, vehicle side slip and lateral acceleration response is shown in Figure 7 . The vehicle lateral motion can be controlled by regulating the steering angle and driving torque based on reference yaw rate input. Additionally, with vehicle speed $=50 \mathrm{~km} / \mathrm{h}$, the host vehicle runs the avoidance manoeuvre through the minimum potential field energy value without lane departure. The generated of desired vehicle lateral motion from artificial potential field approach with application of optimal control theory as well as control system strategy can keep the safety distance margin for collision avoidance.
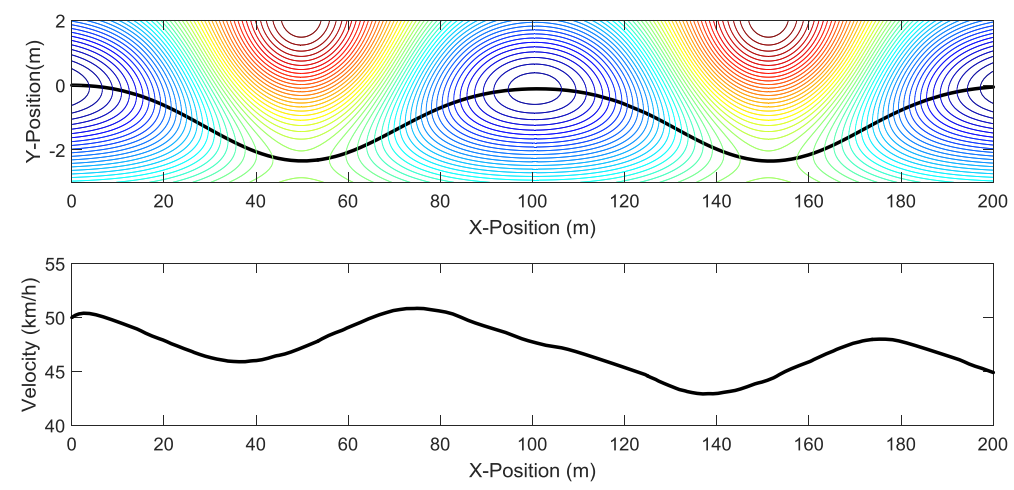

Figure 6. Vehicle trajectory in avoiding obstacles 

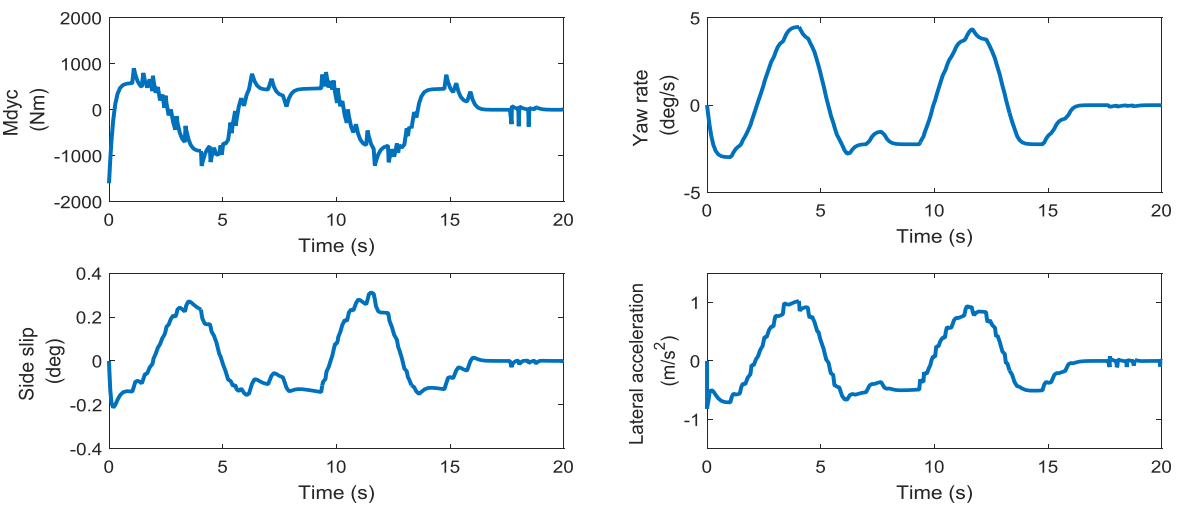

Figure 7. Simulation results of vehicle behaviour.

\section{Conclusion}

In enhancing the performance of active safety, this paper described and studied the motion planning of vehicle obstacle avoidance driver assistance systems based on artificial potential field method. Several potential functions had been developed by considering two stationary obstacles and road edges to describe the risk potential. Lane departure risk potential and collision with obstacle risk potential was considered for control the lateral motion of host vehicle. To complete the collision avoidance execution task, the control system had been formulated based on steering wheel control and DYC. In this work, steering control system was used to complete the steering avoidance manoeuvre while DYC will give the effect to the vehicle speed by control the longitudinal motion of host vehicle. The performance evaluation of proposed collision avoidance driver assistance systems had been investigated through the simulation environment. From simulation results, it can be revealed that, the proposed driving assistance systems for collision avoidance can successfully followed the avoidance manoeuvre with surrounding obstacles. For the future studies, the formulated algorithm can be further explored and expanded into dynamics vehicle collision avoidance environment considering moving obstacles with different driving scenarios.

\section{Acknowledgement}

This work is funded by Ministry of Higher Education, Malaysia under Research University Grant, Universiti Teknologi Malaysia (vote no: 13H73) and Perusahaan Otomobil Nasional (PROTON) Sdn. Bhd (vote no: 4C099). The authors also would like to acknowledge Assoc. Prof. Pongsathorn Raksincharoensak for the valuable knowledge and Smart Mobility Research Centre, Tokyo University of Agriculture and Technology (TUAT) for the placement given during the short-term attachment research programme.

\section{References}

[1] Transport Statistic Malaysia 2014. Ministry of Transportation Malaysia. 2015.

[2] Mohd Yusoff MF, Mohamad NA, Md Nor NG. Malaysian Value of Fatal and Non Fatal Injury due to Road Accident: The Willingness to Pay Using Conjoint Analysis Study. Proceedings of the Eastern Asia Society for Transportation Studies. 2011; 8: 1-13.

[3] Mohd Yusof MF, Md Nor NG, Mohamad NA. Malaysian Value of Statistical Life for Fatal Injury in Road Accident: A Conjoint Analysis Approach. Journal of Society for Transportation and Traffic Studies. 2013; 2(2): 30-40.

[4] Hesse T, Sattel T. An Approach to Integrate Vehicle Dynamics in Motion Planning for Advanced Driver Assistance Systems. IEEE Intelligent Vehicles Symposium. Istanbul. 2007: 1240-1245.

[5] Kamil F, Tang S, Kakhsar W, Zulkifli N, Ahmad SA. A Review on Motion Planning and Obstacle Avoidance Approaches in Dynamic Environments. Advances in Robotics and Automation. 2015; 4(2).

[6] Raja P, Pugazhenthi S. Optimal Path Planning of Mobile Robots: A Review. International Journal of the Physical Sciences. 2012; 7(9): 1314-1320. 
[7] Khatib O. Real Time Obstacle Avoidance for Manipulators and Mobile Robots. International Journal of Robotics and Research. 1986; 5(1): 90-98.

[8] Ge SS, Cui YJ. New Potential Functions for Mobile Robot Path Planning. IEEE Transactions on Robotics and Automation. 2000; 16(5): 615-620.

[9] Bin-qiang Y, Ming-fu Z, Yi W. Research of Path Planning Method for Mobile Robot Based on Artificial Potential Field. 2011 International Conference on Multimedia Technology. Hangzho. 2011: 31923195.

[10] Gerdes JC, Rossetter EJ. A Unified Approach to Driver Assistance Systems Based on Artificial Potential Fields. Journal of Dynamic Systems, Measurement and Control. 2001; 123(3): 431-438.

[11] Wolf MT, Burdick JW. Artificial Potential Functions for Highway Driving with Collision Avoidance. IEEE International Conference on Robotics and Automation. USA. 2008: 3731-3736.

[12] Yin L, Yin Y, Lin CJ. A New Potential Field Method for Mobile Robot Path Planning in Dynamic Environments. Asian Journal of Control. 2009. 11(2): 214-225.

[13] Noto N, Okuda H, Tazaki Y, Suzuki T. Steering Assisting System for Obstacle Avoidance Based on Personalized Potential Field. IEEE Conference on Intelligent Transportation Systems. Alaska. 2012: 1702-1707.

[14] Ma Y, Zheng G, Perruquetti W, Qiu Z. Motion Planning for Non-holonomic Mobile Robots using the iPID Controller and Potential Field. 2014 IEEE/RSJ International Conference on Intelligent Robots and Systems. USA. 2014: 3618-3623.

[15] Noto N, Okuda H, Tazaki Y, Inagaki S, Suzuki T. Obstacle Avoidance Assisting System Based on Personalized Potential Field. SICE Annual Conference 2011. Tokyo. 2011: 476-481.

[16] Hasegawa T, Raksincharoensak P, Nagai M. Risk-potential Based Motion Planning and Control of Proactive Driving Intelligence System for Enhancing Active Safety. Proceedings of International Symposium of Advanced Vehicle Control 2014. Tokyo. 2014.

[17] Raksincharoensak P, Hasegawa T, Nagai M. Motion Planning and Control of Autonomous Driving Intelligence System Based on Risk Potential Optimization Framework. International Journal of Automative Engineering. 2016; 7: 53-60.

[18] Yang X, Wang Z, Peng W. Coordinated Control of AFS and DYC for Vehicle Handling and Stability Based on Optimal Guaranteed Cost Theory. Vehicle System Dynamics. 2009; 7(1): 57-79.

[19] Rieveley RJ, Minaker BP. Variable Torque Distribution Yaw Moment Control for Hybrid Powertrains. SAE Technical Paper Series. 2007; 2007(01-02). 